\title{
Risk stratification in diabetes: Not all patients or perfusion defects are the same
}

\author{
Lawrence H. Young, MD
}

\section{See related article, pp. 1041-1049}

The management of cardiovascular disease in diabetes remains one of the major challenges in contemporary cardiology. In patients without symptomatic or previously documented coronary artery disease (CAD), there is a substantial concern for undetected asymptomatic ischemia ${ }^{1}$ and the risk for developing unheralded myocardial infarction or sudden cardiac death. ${ }^{2}$ When symptomatic CAD is present, there is an even greater concern, because of the potential for advanced atherosclerosis, which carries a very high risk for adverse cardiac outcomes. ${ }^{3}$ In epidemiological studies, it is recognized that the cardiovascular risk of diabetes is related to the duration of diabetes and whether it requires insulin for glycemic control. ${ }^{4}$ In clinical studies, these factors also play into the prognosis of diabetic patients with acute myocardial infarction or those undergoing coronary intervention or bypass surgery. ${ }^{3,5}$

Barmpouletos et $\mathrm{al}^{6}$ herein present the results of a retrospective analysis, suggesting that simple clinical features improve the risk stratification conferred by stress myocardial perfusion imaging in patients with diabetes. These results are potentially highly relevant to the interpretation of test results by nuclear cardiologists. The authors examined the outcomes of 886 diabetic patients studied in a single center for evaluation of suspected or known CAD. Their outcome analysis focused on '"hard events,', specifically including only non-fatal myocardial infarction and cardiac death. During an average follow-up of 2.5 years, 65 patients $(7.3 \%)$ suffered cardiac death and $33(3.7 \%)$ non-fatal MI, indicating a relatively high-risk cohort overall.

From the Section of Cardiovascular Medicine, Yale University School of Medicine, New Haven, CT.

Reprint requests: Lawrence H. Young, MD, Section of Cardiovascular Medicine, Yale University School of Medicine, New Haven, CT 06520; lawrence.young@yale.edu.

J Nucl Cardiol 2010;17:990-2.

1071-3581/\$34.00

Copyright (C) 2010 American Society of Nuclear Cardiology.

doi:10.1007/s 12350-010-9306-3
The study group was fairly typical of a referral population of diabetic patients with known or suspected CAD. Their average age was 61 years, their duration of diabetes averaged 13.2 years, and half were women. Unfortunately, more detailed information on their diabetes was limited because of the retrospective nature of the study. Potentially informative would have been $\mathrm{Hgb}$ A1C levels and the presence of microalbuminuria, renal insufficiency, and diabetic neuropathy. It is worth noting that an additional 109 patients who were not taking diabetes medications were excluded, because of the authors' concern that they might be either non-compliant or erroneously diagnosed. This exclusion resulted in a somewhat higher proportion of insulin-treated patients (55\%) than otherwise might have been anticipated.

On myocardial perfusion imaging, important predictors of adverse cardiac events not surprisingly included summed stress scores $(\mathrm{SSSs})>8$ (present in 192 patients) and gated stress LV ejection fraction $<40 \%$ (present in 115 patients). However, the authors found that age, insulin therapy, and diabetes duration $>10$ years were also independent predictors of cardiac risk. The latter two features are noteworthy in that they are easily obtained in the patient's history and appeared to have an important role in stratifying patients, particularly those with high-risk perfusion abnormalities.

Specifically, patients with SSS $>8$ and diabetes duration $\leq 10$ years vs $>10$ years had significantly lower annual event rates, $4.3 \%$ vs $11 \%$. Those with SSS $>8$ on oral hypoglycemic agents alone vs those on insulin also had significantly lower annual event rates, $3.9 \%$ vs $11.2 \%$. Similar findings were seen when patients with stress $\mathrm{LVEF}<40 \%$ and diabetes duration $\leq 10$ years vs $>10$ years were compared, with annual event rates of $4.3 \%$ vs $14 \%$. The lowest event rates in patients with high-risk perfusion findings were in those with diabetes duration $\leq 10$ years who were also on oral hypoglycemic agents alone. Although only 40 subjects $(21 \%)$ of those with SSS $>8$ fell into this category, there were only three events so that the average annual event rate was just over $2 \%$, rather remarkable for a group of symptomatic patients with moderate-large defects. Similar results were found for patients with an LVEF $<40 \%$ who had shorter durations of diabetes and did not require insulin. Conversely, patients with high-risk perfusion abnormalities or low post-test LVEF, and both long duration 
of diabetes and insulin treatment, were at the highest risk with annualized cardiac event rates of $12 \%-14 \%$.

The authors conclude that diabetes duration and the type of diabetes medication should be incorporated into risk stratification analysis for patients with diabetes referred for stress perfusion imaging. Their data support this conclusion based on the substantial differences in cardiac event rates, depending on the presence or absence of these features. These high-risk diabetes factors can be readily elicited at the time stress testing is performed and should heighten the concern for high-risk perfusion abnormalities.

One of the more remarkable findings of the study is the presence of a relatively low-risk sub-group of patients with high-risk stress perfusion abnormalities. These latter results recall the findings of recent diabetes studies, which have shown surprisingly low cardiac event rates. In the DIAD study of asymptomatic patients with type 2 diabetes, screening myocardial perfusion abnormalities were predictive of cardiac outcomes, but the event rates were quite low; even those patients with moderate-large defects had average annual event rates of only $2.4 \%$ per year. $^{2}$ In ACCORD, a large clinical trial of intensive glycemic control, the annual cardiac event rates were less than $2 \%$ per year in a cohort of over 10,000 patients who had either a prior cardiac event or high-risk for CAD. ${ }^{7}$

While the study by Barmpouletos et al alerts us to the importance of simple historical factors that should be considered in the risk stratification of patients with diabetes referred for cardiac testing, it raises a number of additional questions that warrant consideration. First, are there other diabetes-related factors that might be helpful in stratifying patients referred to nuclear cardiology laboratories? One potentially important and understudied factor is the presence of cardiac autonomic dysfunction. There is increasing evidence that abnormal cardiac autonomic testing is associated with increased cardiac risk, including data from the DIAD study where it was the only factor that predicted both abnormalities on perfusion imaging ${ }^{1}$ and subsequent cardiac events ${ }^{2}$ in asymptomatic patients. Cardiac autonomic dysfunction appears to develop as the result of hyperglycemia and other cardiac risk factors. In addition to serving as an integrated marker of risk, it is possible that autonomic imbalance contributes to the pathophysiology of acute coronary events. Autonomic testing might be useful if used in conjunction with myocardial perfusion imaging, but the approaches to testing still need to be standardized and simplified before it can be used in a routine fashion.

The identification of a sub-group of patients, with known or suspected CAD and traditionally high-risk perfusion abnormalities, who have reasonably low event rates, raises a provocative clinical question regarding their management. Can such patients be treated safely with aggressive medical therapy rather than coronary intervention? Given the relatively small number of patients in this sub-group, it would be important for other laboratories to determine whether they share this same observation. If confirmed, then there might be a reason to actually test a conservative vs interventional approach, to determine whether coronary revascularization reduces cardiac events in this sub-group. One point worth noting is that 90 patients had early coronary intervention after stress testing and were excluded from the analysis. It is possible that the outcome rates might have been higher had all these patients been managed with a conservative strategy.

The results of this study also raise the important pathophysiological question of why the duration of diabetes or the requirement for insulin influence the incidence of cardiac events in patients with the same degree of stress perfusion abnormality or post-stress LVEF. It is reasonable to suspect that some combination of plaque vulnerability and/or thrombotic tendency might contribute to their increased cardiovascular risk, but the critical factors are still not well understood. The authors suggest that more severe atherosclerosis and the potential lack of collaterals might also contribute to the high risk in patients with more long-standing or severe diabetes. The results of this study do not identify pathophysiological mechanisms, but they do highlight the need for further research to delineate these mechanisms and hopefully to develop more effective therapeutic approaches to prevention in these high-risk patients.

Thus, the findings of Barmpouletos et al remind us that not all patients with diabetes are the same and also inform us that not all perfusion defects carry the same risk for adverse cardiac events. As a practical point, they should alert cardiologists to consider simple diabetesrelated historical features when providing risk stratification for patients referred to the nuclear cardiology laboratory.

\section{References}

1. Wackers FJ, Young LH, Inzucchi SE, Chyun DA, Davey JA, Barrett EJ, Taillefer R, Wittlin SD, Heller GV, Filipchuk N, et al. Detection of silent myocardial ischemia in asymptomatic diabetic subjects: The DIAD study. Diabetes Care 2004;27:1954-61.

2. Young LH, Wackers FJ, Chyun DA, Davey JA, Barrett EJ, Taillefer $\mathrm{R}$, Heller GV, Iskandrian AE, Wittlin SD, Filipchuk N, et al. Cardiac outcomes after screening for asymptomatic coronary artery disease in patients with type 2 diabetes: The DIAD study: A randomized controlled trial. JAMA 2009;301:1547-55.

3. BARI Investigators. Influence of diabetes on 5-year mortality and morbidity in a randomized trial comparing CABG and PTCA in 
patients with multivessel disease: The Bypass Angioplasty Revascularization Investigation (BARI). Circulation 1997;96: 1761-9.

4. Fox C, Sullivan L, D'Agostino RS. The significant effect of diabetes duration on coronary heart disease mortality: The Framingham Heart Study. Diabetes Care 2004;27:704-8.

5. Brooks MM, Jones RH, Bach RG, Chaitman BR, Kern MJ, Orszulak TA, Follmann D, Sopko G, Blackstone EH, Califf RM Predictors of mortality and mortality from cardiac causes in the bypass angioplasty revascularization investigation (BARI) randomized trial and registry. For the BARI Investigators. Circulation 2000;101:2682-9.

6. Barmpouletos D, Stavens G, Ahlberg AW, Katten DM, O'Sullivan DM, Heller GV. Duration and type of therapy for diabetes: Impact on cardiac risk stratification with stress electrocardiographic-gated SPECT myocardial perfusion imaging. J Nucl Cardiol 2010. doi: 10.1007/s12350-010-9293-4.

7. The Action to Control Cardiovascular Risk in Diabetes Study Group. Effects of intensive glucose lowering in type 2 diabetes. NEJM 2008;258:2545-59. 